\title{
RCAN1-4 is a thyroid cancer growth and metastasis suppressor
}

\author{
Chaojie Wang, ${ }^{1,2}$ Motoyasu Saji, ${ }^{1}$ Steven E. Justiniano, ${ }^{1}$ Adlina Mohd Yusof, ${ }^{1}$ Xiaoli Zhang, ${ }^{3}$ \\ Lianbo Yu, ${ }^{3}$ Soledad Fernández, ${ }^{3}$ Paul Wakely Jr., ${ }^{4}$ Krista La Perle, ${ }^{5}$ Hiroshi Nakanishi, ${ }^{1}$ \\ Neal Pohlman, ${ }^{1}$ and Matthew D. Ringel ${ }^{1,2}$ \\ DDivision of Endocrinology, Diabetes and Metabolism, Department of Internal Medicine, ${ }^{2}$ Ohio State Biochemistry Program, \\ ${ }^{3}$ Center for Biostatistics, Department of Biomedical Informatics, ${ }^{4}$ Department of Pathology, ${ }^{5}$ Department of Veterinary \\ Biosciences and Comparative Pathology and Mouse Phenotyping Shared Resource, The Ohio State University and Arthur \\ G. James Comprehensive Cancer Center, Columbus, Ohio, USA.
}

\begin{abstract}
Metastasis suppressors are key regulators of tumor growth, invasion, and metastases. Loss of metastasis suppressors has been associated with aggressive tumor behaviors and metastatic progression. We previously showed that regulator of calcineurin 1, isoform 4 (RCAN1-4) was upregulated by the KiSS1 metastatic suppression pathway and could inhibit cell motility when overexpressed in cancer cells. To test the effects of endogenous RCAN1-4 loss on thyroid cancer in vivo, we developed RCAN1-4 knockdown stable cells. Subcutaneous xenograft models demonstrated that RCAN1-4 knockdown promotes tumor growth. Intravenous metastasis models demonstrated that RCAN1-4 loss promotes tumor metastases to the lungs and their subsequent growth. Finally, stable induction of RCAN1-4 expression reduced thyroid cancer cell growth and invasion. Microarray analysis predicted that nuclear factor, erythroid 2-like 3 (NFE2L3) was a pivotal downstream effector of RCAN1-4. NFE2L3 overexpression was shown to be necessary for RCAN1-4-mediated enhanced growth and invasiveness and NEF2L3 overexpression independently increased cell invasion. In human samples, NFE2L3 was overexpressed in TCCA thyroid cancer samples versus normal tissues and NFE2L3 overexpression was demonstrated in distant metastasis samples from thyroid cancer patients. In conclusion, we provide the first evidence to our knowledge that RCAN1-4 is a growth and metastasis suppressor in vivo and that it functions in part through NFE2L3.
\end{abstract}

Conflict of interest: The authors have declared that no conflict of interest exists.

Submitted: September 12, 2016 Accepted: January 24, 2017 Published: March 9, 2017

Reference information: JCI Insight. 2017;2(5):e90651. https:// doi.org/10.1172/jii.insight.90651.

\section{Introduction}

Metastasis is a complex process by which cancer cells spread to distant locations; it requires individual or groups of cells to locally invade, intravasate, survive in circulation, extravasate, and grow and, in some cases, invade at metastatic sites (1). Metastasis suppressors are proteins that inhibit any step of metastasis without affecting primary tumor formation (2). Since Steeg et al. described the first metastasis suppressor gene, NM23 (3), more than twenty metastasis suppressor genes have been identified, with varying degrees of evidence to support their functions (4-6). Metastasis suppressors have been shown to play pivotal roles in restraining tumor cells from disseminating into metastatic sites, and their expression and/or function is typically reduced during metastatic progression (7-9).

Metastatic dormancy refers to the ability of metastatic cancer cells to survive but not grow and progress at the metastatic sites (10). Thyroid cancer is a relatively indolent tumor when it is well differentiated, even after it has metastasized to the lungs, the most common site of distant spread (11). Because of this indolent nature of even metastatic lesions, thyroid cancer is an excellent model to study the mechanisms of metastatic dormancy. Clinically, the loss of metastatic dormancy can occur in patients with thyroid cancer, and a late-stage aggressive course can occur, resulting in cancer-related death $(12,13)$. Thus, in addition to being an excellent model of dormancy, thyroid cancer is an excellent model to study the defining factors that regulate "the switch" from dormancy to progression, which is also crucial for defining new targets for thyroid cancer therapy and/or identifying markers for tumors likely to progress more rapidly.

Several studies have demonstrated that individuals with Down's syndrome that have trisomy 21 have a reduced incidence of solid tumors compared with the normal population (14-17). Regulator of calcineurin 1 (RCAN1, also known as Down's syndrome critical region 1 [DSCR1]) is one of the genes 
on chromosome 21 that contributes to this tumor protective effect (18). RCAN1 is a gene with multiple transcriptional start sites located on chromosome 21 within the Down's syndrome critical region that expresses two main isoforms, RCAN1-1 and RCAN1-4, depending on the promoter that is utilized (19). While RCAN1-1 is constitutively expressed, RCAN1-4 expression is induced in response to various physiological changes (20). RCAN1-4 is a competitive inhibitor for the phosphatase calcineurin (21) and thereby suppresses calcineurin-mediated dephosphorylation and activation of nuclear factor of activated T cells (NFAT) (22). Since NFATs are primary transcription activators for the RCAN1-4 gene, RCAN1-4 serves as a negative feedback regulator of calcineurin/NFAT signaling.

NFATs had been reported to regulate multiple events during cancer progression, including cell invasion, motility, and angiogenesis (23). RCAN1-4 has been reported to suppress endothelial cell migration, neovascularization, and tumor growth with reduced vascularity through inhibition of NFAT activity, suggesting a role for RCAN1 in negative regulation of tumor angiogenesis $(24,25)$. Indeed, Baek et al. demonstrated that loss of all RCAN1 isoforms reversed this tumor growth suppression effect in a Down's syndrome mouse model, and one extra copy of RCAN1-4 could inhibit tumor growth with decreased angiogenesis (18). In addition, RCAN1-4, rather than RCAN1-1, was shown to be selectively expressed in the endothelium during development and in B16-F1 and Lewis lung carcinoma xenografts, further supporting a role for RCAN1-4 in the tumor microenvironment in Down's syndrome, particularly in endothelial cells (26).

In addition to this effect in Down's syndrome, a role for RCAN1 has also been demonstrated in sporadic tumors. It has been reported that RCAN1 loss is prevalent across tumor types that are commonly reduced in patients with Down's syndrome, suggesting a tumor-inhibitory role for the protein in cancers (27). In addition, it has been demonstrated that the treatment of cells with the metastasis suppressor metastin (KiSS1 gene product) results in a marked increase in RCAN1-4 gene and protein expression and that its ability to reduce cell motility is dependent on RCAN1-4 (28). In human papillary thyroid cancers (PTC), RCAN1 expression is increased in primary tumors versus normal tissue, but the expression is lost in metastases, a pattern consistent with a metastasis suppressor (28). Further studies determined that overexpression of RCAN1-4 in cancer cells inhibits cell motility and that loss of endogenous RCAN1-4 promoted cell migration in vitro. Finally, it has been shown that RCAN1-4 protein stability is reduced in some cancer cell lines characterized by high degrees of motility in vitro (29).

The objective of the present study is to investigate the cancer cell-autonomous function of RCAN1-4 on thyroid cancer growth and metastasis progression in vivo. Using thyroid cancer cell lines with stable reduction of RCAN1-4 levels, we demonstrate that loss of endogenous RCAN1-4 increases cell growth in 3D conditions and invasion through Matrigel in vitro, promotes tumor growth in flank xenograft model, and enhances tumor metastasis to lungs following tail vein injection. In contrast, induced expression of RCAN1-4 reduced 3D growth and invasion. We also report that nuclear factor, erythroid 2-like 3 (NFE2L3) is responsible in part for these RCAN1-4-mediated effects and independently enhances cancer cell invasion and growth and that it is overexpressed in human thyroid cancer tissues. These data identify cancer cell RCAN1-4 as a regulator of tumor growth and metastasis in vivo, report a potentially previously undescribed mechanistic role for NFE2L3 in RCAN1-4 effects, and suggest that NFE2L3 may be an important regulator of cancer progression.

\section{Results}

Establishment of stable RCAN1-4 knockdown cell lines. To assess levels of endogenous RCAN1-4 in thyroid cancer cells, we screened a panel of confirmed human thyroid cancer cell lines with stable expression of luciferase for subsequent in vivo imaging by Western blot. While all cell lines had similar RCAN1-1 expression (Figure 1A), FTC236 cells and HTh74 cells had higher endogenous RCAN1-4 expression versus the other cell lines (Figure 1A). These two cell lines were chosen to create stable RCAN1-4 knockdown cells using shRNA specifically targeting RCAN1-4 (shRCAN1-4). Western blot demonstrated that both FTC236 and HTh74 shRCAN1-4 clones had more than 80\% knockdown of RCAN1-4 compared with nontargeting scrambled control shRNA-transfected stable clones (shCtrl). RCAN1-1 expression was not altered, and, therefore, RCAN1-1 was able to serve as a control protein to evaluate the specificity of the shRNA (Figure 1B). These cell lines were selected for subsequent in vivo studies.

RCAN1-4 knockdown promotes $3 D$ cell growth in vitro. To examine whether loss of RCAN1-4 affected cell proliferation in 2D growth conditions, shCtrl and shRCAN1-4 cells were seeded in 6-well plates and subjected to cell proliferation assays. In both FTC236 and HTh74 cells, shCtrl and shRCAN1-4 cells had no significant difference in cell proliferation over a 3-day period (Figure 1C). These data are similar to prior work using 
A

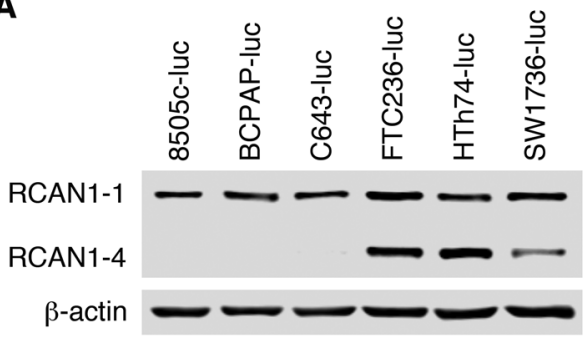

B

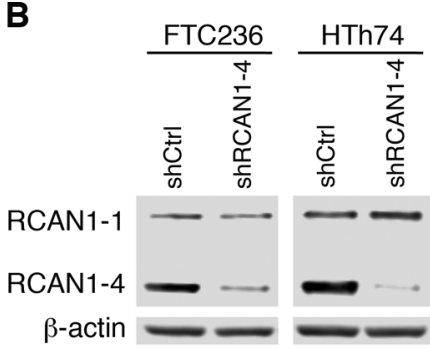

C

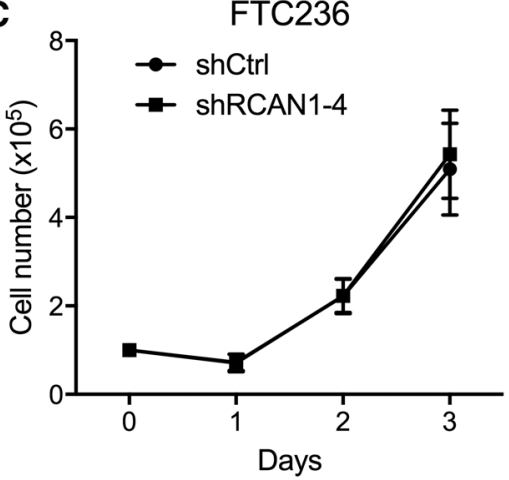

HTh74

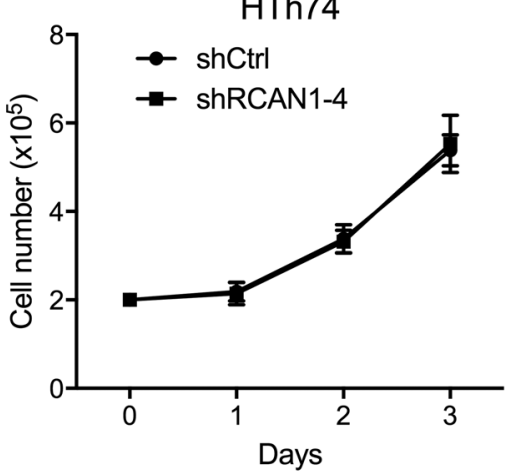

D
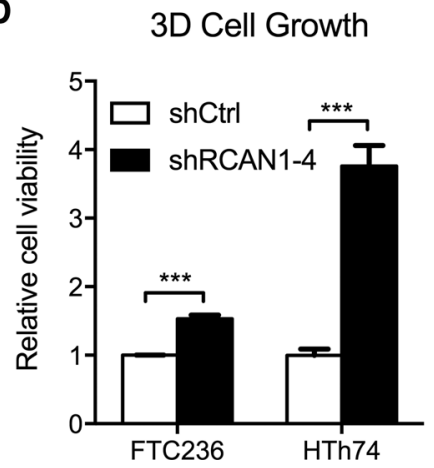

Figure 1. RCAN1-4 knockdown promotes cell 3D spheroid growth in vitro. (A) Endogenous RCAN1-4 expression was analyzed by Western blot in 6 established thyroid cancer cell lines stably expressing luciferase. (B) RCAN1-4 knockdown efficiency of shRCAN1-4 stable cells was confirmed using Western blot. (C) Cells were seeded in 6-well plates in triplicate, and viable cells were counted at 24,48 , and 72 hours after seeding. Each data point represents the mean \pm standard error of 3 independent experiments. (D) Cells were seeded on Corning spheroid 96-well microplates and incubated for 2 or 3 days for FTC236 and HTh74 cells, respectively. Cell viability was determined; the data were normalized to the corresponding shCtrl cells. Error bars represent SEM. A linear mixed model was used to determine the statistical significance. ${ }^{* *} P<0.001$.

transient siRNA in thyroid cancer cells (29). To determine whether RCAN1-4 knockdown affected 3D cell growth in vitro, cells were seeded in spheroid microplates. For FTC236 cells, spheroids that grow vertically were identified in the shRCAN1-4 cells, while no obvious spheroids were observed from the shCtrl cells (data not shown). In HTh74 cells, shRCAN1-4 cells formed larger spheroids compared with the shCtrl cells (data not shown). To quantify growth in $3 \mathrm{D}$ conditions, cell viability assays were performed and confirmed that shRCAN1-4 cell viability was significantly higher than shCtrl cell viability for both cell lines $(P<0.001$ for both, Figure 1D). Thus, loss of RCAN1-4 resulted in increased cell viability in 3D but not 2D conditions.

RCAN1-4 knockdown promotes cancer cell invasion in vitro. We next evaluated the effects of RCAN1-4 knockdown on invasion through Matrigel in vitro using coated Transwell filters. Representative images are shown in Figure 2A, and the number of invaded cells per field was counted for quantitation. The mean number of invaded cells was enhanced more than 3-fold in FTC236 cells and 2-fold in HTh74 cells by RCAN1-4 knockdown versus control cells ( $P<0.001$, Figure $2 \mathrm{~B})$.

RCAN1-4 modulates tumor growth in vivo. We next investigated the role of endogenous RCAN1-4 in tumor growth in vivo. The shCtrl and shRCAN1-4 cells from both cell lines were subcutaneously injection in athymic nude mouse flanks. Tumor growth was monitored using both an In Vivo Imaging System (IVIS) and caliper measurements over 10 to 12 weeks based on the size of the tumors and removal criteria for the mice. For both FTC236 and HTh74 cells, IVIS imaging demonstrated that shRCAN1-4 xenografts had stronger bioluminescence signals compared with corresponding shCtrl xenografts (Figure 3A). In the FTC236 group, a difference in tumor volumes between the shCtrl and shRCAN1-4 xenografts was not observed until the eighth week. The tumor volumes were significantly different through the end of the study at the tenth week $(n=9 ; P=0.0087)$. 
A

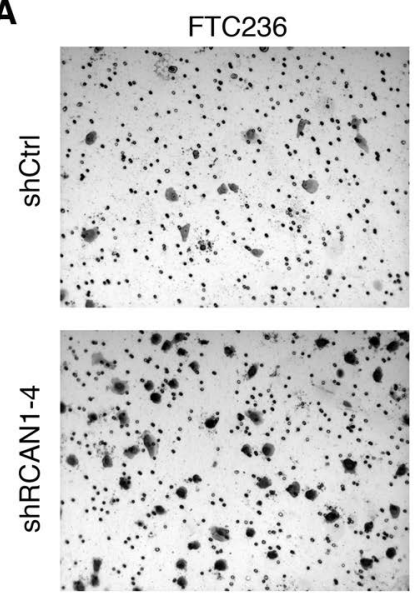

B

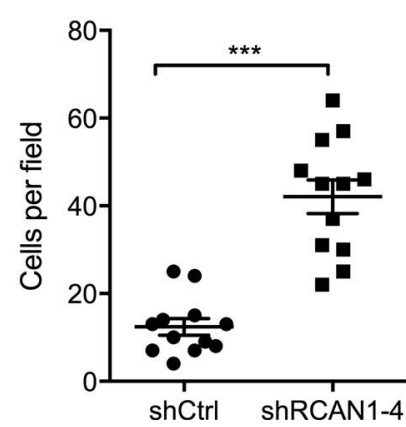

HTh74
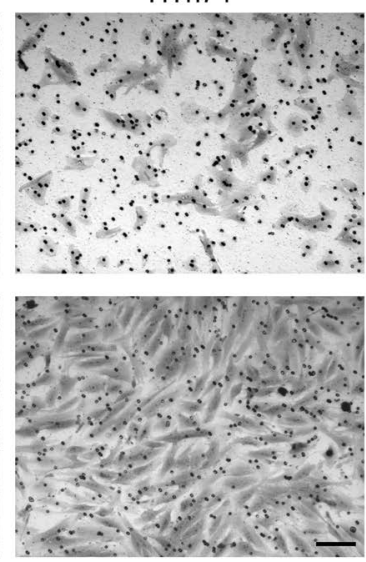

HTh74

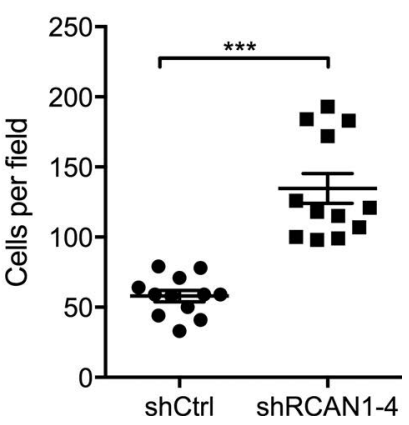

Figure 2. RCAN1-4 knockdown promotes cancer cell invasion in vitro. (A) shCtrl and shRCAN1-4 cells were seeded on Matrigel-coated 8- $\mu \mathrm{m}$ pore invasion membranes and incubated with gradient medium (FTC236: 1\% gradient for 24 hours; HTh74: $10 \%$ gradient for 36 hours). Representative images of the invaded cells are shown. Scale bar: $100 \mu \mathrm{m}$. (B) The number of invaded cells was counted for each image field using ImageJ software. Data represent the mean \pm SEM of 3 independent experiments, with 4 fields counted per experiment. A linear mixed model was used to determine the statistical significance. ${ }^{* *} P<0.001$.

The final average tumor volumes were $2,360.4 \mathrm{~mm}^{3}$ for FTC236 shCtrl cells and 4,571.8 $\mathrm{mm}^{3}$ for FTC236 shRCAN1-4 cells (Figure 3B). In the HTh74 group, a difference in tumor volume between the shCtrl and shRCAN1-4 xenografts was observed starting from the seventh week, and the growth rate was also significantly different through end of study at the twelfth week $(n=7 ; P<0.0001)$. The final average tumor volumes were $172.4 \mathrm{~mm}^{3}$ for HTh74 shCtrl cells compared with 2,514 $\mathrm{mm}^{3}$ for HTh74 shRCAN1-4 cells (Figure 3B). Tumor sections were subjected to Ki-67 staining to assess in vivo cell proliferation. In HTh74 cells, shRCAN1-4 tumors had significantly more Ki-67-positive cells compared with shCtrl tumors $(P<0.001$, Figure 3, C and D). Although the number of FTC236 cells demonstrating Ki-67 immunoreactivity was not significantly different, shRCAN1-4 tumors exhibited more intense Ki-67 staining compared with shCtrl tumors (55.61\% versus $47.97 \%$ ) (Figure 3, C and D). To determine whether apoptosis was also regulated in vivo, cleaved caspase-3 staining was measured. No significant changes were observed in cleaved caspase-3-positive cells (Figure 3, C and D). To assess differences in tumor vascularity, CD31 staining was performed. A significant increase in the number of intratumoral microvessels was identified in the HTh74 shRCAN1-4 tumors compared with the HTh74 shCtrl tumors $(P<0.001$, Supplemental Figure 1; supplemental material available online with this article; https://doi.org/10.1172/jci.insight.90651DS1). By contrast, no difference in CD31 staining was observed in the FTC236 xenografts. No difference was observed in tumor-associated macrophages, as determined by F4/80 staining, for either tumor xenograft (Supplemental Figure 1). In conclusion, the increased subcutaneous xenograft growth in vivo is associated with increased cell proliferation and angiogenesis (in HTh74 tumors) but not reduced apoptosis.

RCAN1-4 regulates tumor metastasis in vivo. We next investigated whether RCAN1-4 in cancer cells regulates metastatic implantation and/or growth in vivo using a tail vein model. The shCtrl and shRCAN1-4 cells were injected into the tail veins of athymic nude mice. Successful injection was verified by immediate IVIS imaging to confirm signal in the lungs of each mouse (data not shown). Subsequent metastasis colonization and growth were monitored weekly using IVIS imaging. In the FTC236 group, 80\% of the mice injected with shRCAN1-4 cells had strong lung bioluminescence signals at the end of the study, whereas only $10 \%$ of the mice injected with shCtrl cells had weak lung bioluminescence signals (Figure $4 \mathrm{~A}$ ). For the HTh74 cells, $41.67 \%$ of the mice in the shRCAN1-4 group had lung metastasis signal 3 weeks after injection, while none of the mice in the shCtrl group had any metastasis signal at the same time point. After 12 weeks, $75 \%$ of the mice in the shRCAN1-4 group had lung metastasis signals, while for the shCtrl group, only $7 \%$ of the mice showed metastasis signal (Figure $4 \mathrm{~A}$ ). Whole body bioluminescence signals were quantified at the end of the study and statistically analyzed. For both FTC236 and HTh74 cell lines, the shRCAN1-4 group had significantly stronger bioluminescence signals compared with the $\operatorname{sh} \operatorname{ctrl}$ group $(n=$ 10-14, $P<0.001$ ) (Figure 4B). The mice were dissected, and the lungs were fixed and stained with H\&E. Histology examination confirmed the higher frequency of lung metastases in shRCAN1-4 cells versus shCtrl cells in the IVIS imaging results. Representative histology images are shown in Figure 4C.

RCAN1-4 induction reduces thyroid cancer cell growth and invasion. To determine if RCAN1-4 induction reduces thyroid cancer cell growth or invasion, two cell lines with low basal RCAN1-4 expression, 8505c rtTA TRE-RCAN1-4 cells and C643 rtTA TRE-RCAN1-4 cells, were created and treated with vehicle control or $0.5 \mu \mathrm{g} / \mathrm{ml}$ doxycycline for 24 hours to confirm that induction of RCAN1-4 was achieved (Fig- 
A

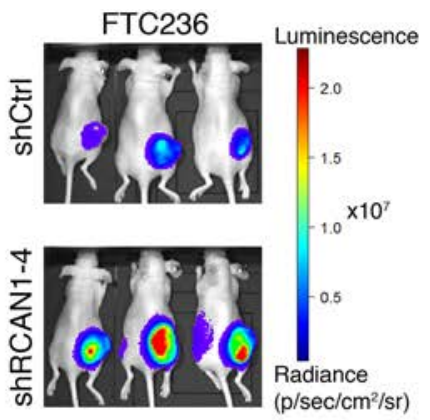

B
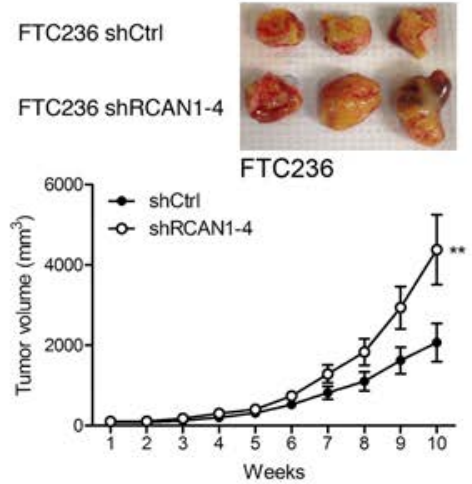

C

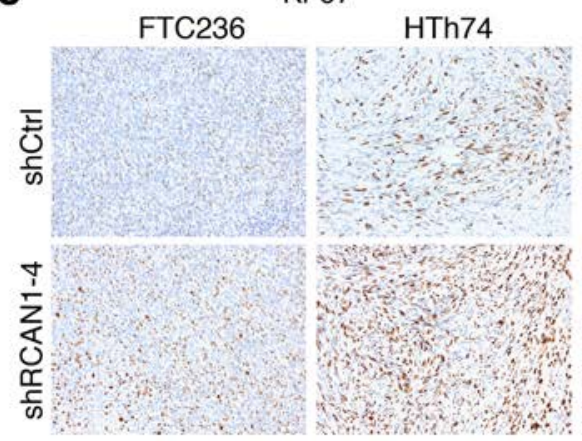

D

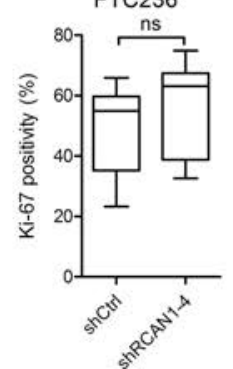

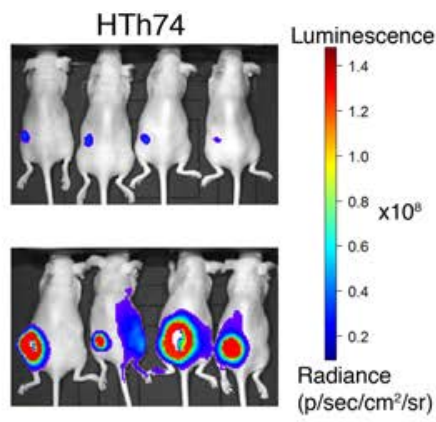

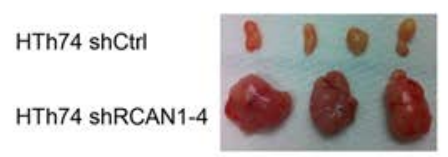

HTh74

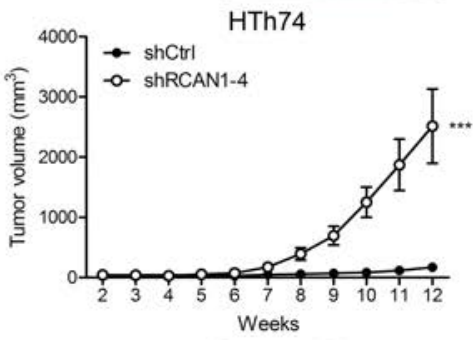

Cleaved Caspase-3

HTh74
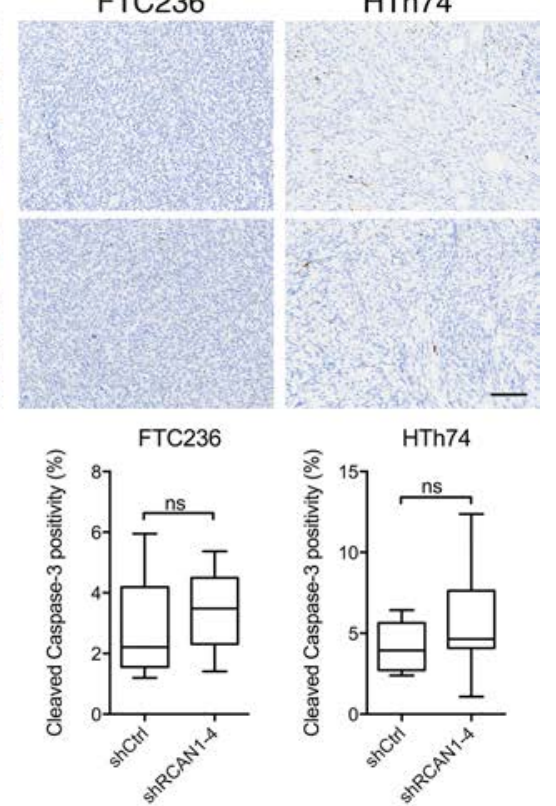

Figure 3. RCAN1-4 knockdown promotes tumor growth in vivo. (A) shCtrl and shRCAN1-4 cells $(1 \times$ ${ }^{10^{6}}$ ) were injected into athymic nude mouse flanks. Tumor size was monitored by IVIS and caliper measurements. Representative bioluminescence images are shown. (B) Top: isolated FTC236 and HTh74 tumors after dissection at the end of the study (10 and 12 weeks, respectively). Bottom: tumor growth curves based on caliper measurements. Each data point is the mean tumor volume \pm SEM. (C) Representative images of $\mathrm{Ki}-67$ and cleaved caspase-3 staining for the tumors. Scale bar: $100 \mu \mathrm{m}$. (D) Box plots of Ki-67 staining (left) and cleaved caspase-3 staining (right) for the tumors. Ten random fields were quantified and averaged for each animal. The horizontal line in the box represents the median. The box boundaries are the Q1 (25\%) and Q3 (75\%), respectively. Linear mixed models were used to perform all the statistical analyses $(n=7-9) .{ }^{*} P<$ $0.01,{ }^{* * *} P<0.001$.

ure 5). The doxycycline-treated cells were subjected to spheroid growth and invasion assays. 8505c-rtTA and C643-rtTA cells without the tetracycline response element treated with doxycycline were used as a control to account for potential doxycycline effects on spheroid growth and invasion. In both cell lines, induction of RCAN1-4 reduced 3D growth and Matrigel invasion (Figure 5).

NFE2L3 has a functional role in RCAN1-4regulated invasion. To investigate the effects of RCAN1-4 knockdown in tumor cells on gene transcription, microarray analysis was performed using the Affymetrix Human Transcriptome Array 2.0 (HTA 2.0) platform with RNA isolated from shCtrl and shRCAN1-4 cells for the FTC236 and HTh74 cell lines. For both cell lines, differentially expressed genes were defined using $P<0.001$ and fold change $>2.0$. Heatmaps of the differentially expressed genes were created (Figure 6A), and principal component analysis (PCA) was performed to identify gene panels that best distinguish shRCAN1-4 cells from shCtrl cells (Supplemental Figure 2). Altered expression of the genes

included in the PCA groups was confirmed by quantitative reverse transcription PCR (qRT-PCR) (Figure 6B). Interestingly, the differentially expressed genes in the PCA for FTC236 and HTh74 cells did not overlap, indicating that the two cell lines may be regulated by RCAN1-4 via distinct mechanisms. To determine whether any of the genes were overexpressed or underexpressed consistently in both shRCAN1-4 cell lines, we performed additional qRT-PCR experiments for the PCA-identified mRNAs. Of the top 3 overexpressed genes for each cell line, NFE2L3 was the most consistent between both cell lines, with a 21.56-fold increase in the HTh74 shRCAN1-4 cells and a 1.96-fold increase in the FTC236 shRCAN1-4 cells (Supplemental Figure 3). In addition, NFE2L3 was the most overexpressed gene in the HTh74 shRCAN1-4 cell microarray (69-fold increase). Thus, NFE2L3 was chosen for further analysis.

NFE2L3 mRNA and protein overexpression was confirmed to be higher in the shRCAN1-4 cells versus the shCtrl cells by qRT-PCR and Western blot in both cell lines. HEK293 cells transfected with NFE2L3 
A

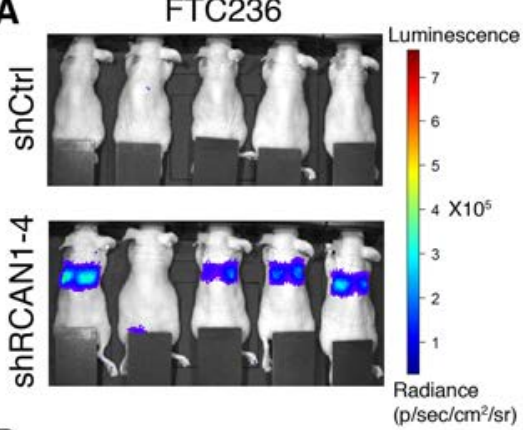

B

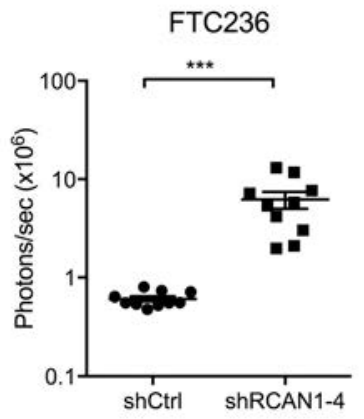

C

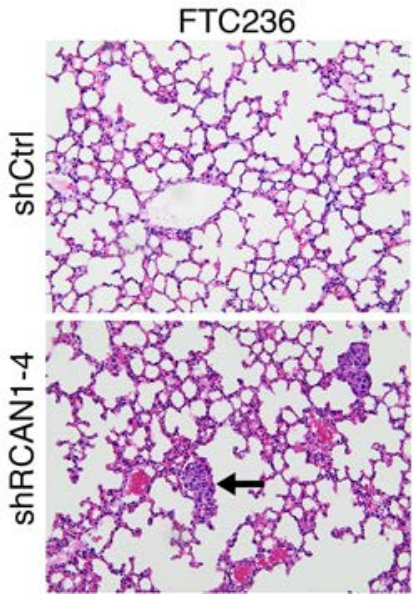

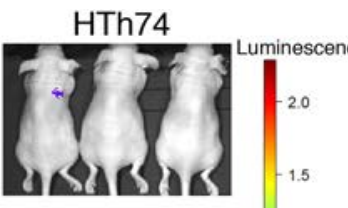

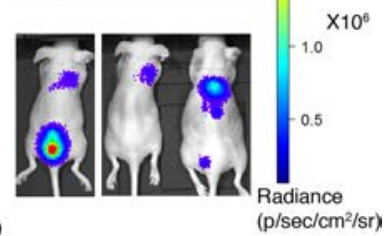

Figure 4. RCAN1-4 knockdown promotes metastasis. (A) FTC236 and HTh74 cells with stable shCtrl or shRCAN1-4 expression were injected into athymic nude mice through tail veins. Tumor metastases and growth were monitored using IVIS imaging. Representative images of mouse whole body bioluminescence at 5 weeks for FTC236 cells and 12 weeks for HTh74 cells are shown. (B) Mouse whole body bioluminescence at the end of the study was quantified. Data points represent bioluminescence activity for each individual animal. The horizontal line represents the mean and the error bars represent the SEM $(n=10-14)$. A linear mixed model was used to determine the statistical significance. ${ }^{* * *} P<0.001$. (C) Representative images of $\mathrm{H} \& \mathrm{E}$-stained lungs with metastases (arrows). Scale bar: $100 \mu \mathrm{m}$.
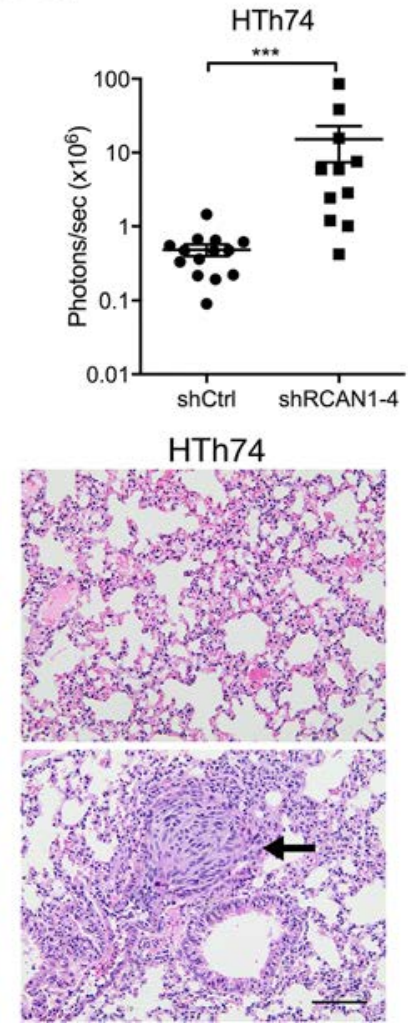

plasmid were used as the control, which had been reported to express 3 forms of NFE2L3 due to posttranslational regulation (30) (Figure 6, C and D). To investigate whether NFE2L3 is upregulated in the xenografts in vivo, we also performed immunohistochemistry (IHC) staining of the xenograft tumor and lung metastasis sections using NFE2L3 antibody. NFE2L3 overexpression was observed in shRCAN1-4 tumors and lung metastases (Figure 6, E and F). The level of overexpression was more pronounced in the HTh74 tumors, which is consistent with the in vitro data. Thus, NFE2L3 expression is increased with loss of RCAN1-4 in vitro and in vivo, and the effect is greater in the HTh74 cells. Finally, in a panel of thyroid cancer cell lines, the cell lines with the highest levels of NFE2L3 had low levels of RCAN1-4, while those with the highest levels of RCAN1-4 had low levels of NFE2L3 (Supplemental Figure 4), although the inverse correlation was not seen in all cell lines tested.

To determine whether NFE2L3 is mechanistically involved in RCAN1-4 function in cancer cells, we performed both 3D spheroid growth assays and invasion assays that are regulated by RCAN1-4 in vitro (Figures 1 and 2). NFE2L3 was knocked down using NFE2L3 specific siRNA (siNFE2L3) in the FTC236 and HTh74 shRCAN1-4 cells that express NFE2L3 basally. The knockdown efficiency was confirmed to be $>90 \%$ as determined by mRNA level using qRT-PCR in both cell lines

(Figure 7A), and protein loss was confirmed by Western blot (Figure 7B). The cells were then subjected to 3D spheroid growth assay. Loss of NFE2L3 in both FTC236 and HTh74 shRCAN1-4 cells decreased spheroid formation and growth (Figure 7C). Matrigel invasion assays demonstrated that loss of NFE2L3 reduced invasiveness compared with control-transfected cells (Figure 7, D and E). These data indicate that NFE2L3 is involved in RCAN1-4-regulated 3D cell growth and invasion.

To assess whether NFE2L3 regulates cell growth and invasion independently, the shCtrl stable cells with no manipulation of RCAN1-4 expression were transfected with NFE2L3 expression vector. Overexpression of NFE2L3 was confirmed by Western blot (Figure 8A). The 3D spheroid assay found no growth difference between NFE2L3-transefected cells and control-transfected cells (Supplemental Figure 5). For Matrigel invasion assays, in FTC236 and HTh74 cells, NFE2L3 overexpression significantly increased cell invasion (Figure $8, \mathrm{~B}$ and C, $P<0.001$ ).

In conclusion, NFE2L3 is an important downstream effector of RCAN1-4-regulated tumor growth and metastasis. However, there may be other important players in this process. The array data showed 8 genes that are commonly regulated by RCAN1-4 knockdown in both cell lines (with fold change $>2$ and $P<0.001$ ): EPHA7, FRMD4A, SERPINE1, SLCO1B3, SOX9, STC1, TNFRSF9, and TSPAN15. Studies on the functions of these genes are ongoing.

NFE2L3 is overexpressed in clinical cancer samples. To determine whether NFE2L3 overexpression correlates with thyroid cancer in humans, we examined NFE2L3 expression in 503 tumor samples and 59 
A

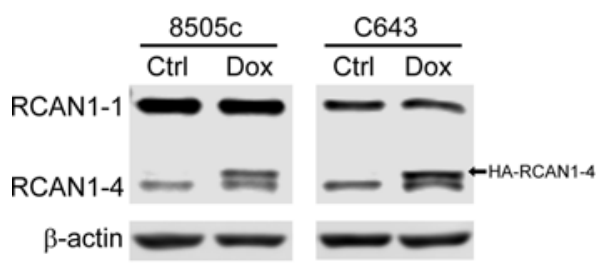

C

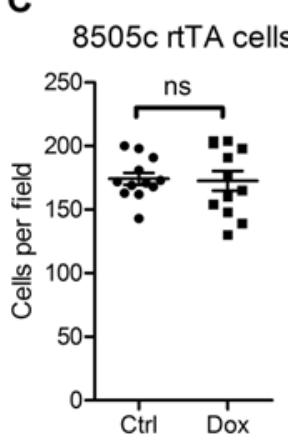

B

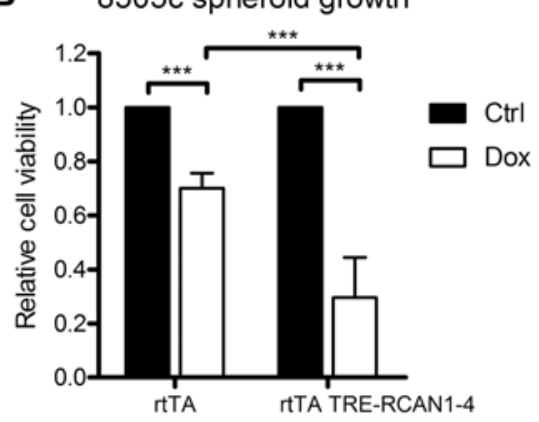

D

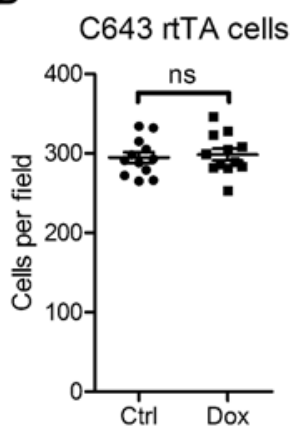

C643 spheroid growth

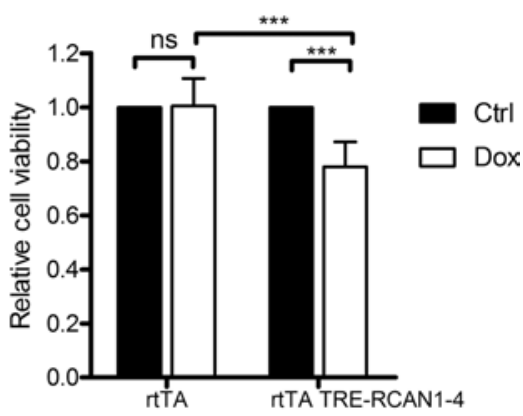

C643 rtTA

TRE-RCAN1-4 cells

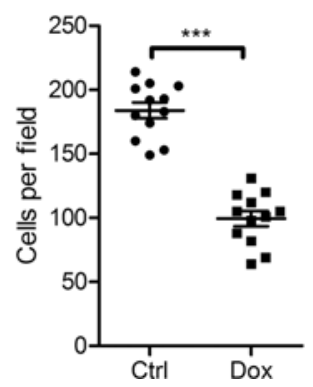

Figure 5. RCAN1-4 overexpression suppresses cell spheroid growth and invasion in vitro. 8505c rtTA TRE-RCAN1-4 cells and C643 rtTA TRE-RCAN1-4 cells were treated with control (Ctrl) or $0.5 \mu \mathrm{g} / \mathrm{ml}$ doxycycline (Dox) for 24 hours. (A) Induction of RCAN1-4 expression was analyzed by Western blot in these treated cells. Actin was the loading Ctrl. The induced RCAN1-4 was HA tagged (HA-RCAN1-4). (B) The Dox-treated cells were subjected to spheroid growth assay. 8505c-rtTA and C643-rtTA parental cells were used as a Ctrl for Dox effects on spheroid growth. The data were normalized to the Ctrl-treated cells. (C and $\mathbf{D})$ The Dox-treated cells were subjected to Matrigel invasion assay. The number of invaded cells was counted for each image field. Each dot is a data point. The horizontal line represents the mean and the error bars represent the SEM. The rtTA parental cells treated with Dox also were used as a Ctrl for Dox effects on cell invasion. A linear mixed model was used to determine the statistical significance. ${ }^{* * *} P<0.001$.

normal samples from The Cancer Genome Atlas (TCGA) thyroid carcinoma cohort (31, 32). NFE2L3 expression in the tumor samples was 7.52-fold higher than that of normal tissue samples, which is statistically significant $(P<2.2 \mathrm{e}-16)$ (Figure 9A). In this cohort, there was a statistically significant but modest inverse relationship between RCAN1 (both isoforms) and NFE2L3 expression $(\mathrm{R}=-0.31 ; \mathrm{P}<0.001)$. The depth of sequencing does not distinguish between the two isoforms, making it not possible to directly test for an inverse relationship between RCAN1-4 and NFE2L3 in this data set. To further explore the clinical relevance of NFE2L3 expression in thyroid cancer, we performed IHC staining using distant metastatic thyroid cancer samples, with paired primary thyroid cancer and normal thyroid samples when available. Samples from 15 cases were stained, including 9 PTC, 5 follicular thyroid cancers (FTC), and 1 anaplastic thyroid cancer (ATC). From those cases, 12 also had metastatic samples from various sites, including lung, lymph node, and bone. Ten cases had paired normal thyroid samples, and eleven of the fifteen had samples from both the central regions and invasive fronts of the primary tumors. Details regarding pathological features and tumor locations are provided in Supplemental Table 1. In general, NFE2L3 expression was lowest in most normal tissues, with increasing expression in the primary tumors, and highest expression in the invasive fronts and metastatic lesions (Figure 9, B and C). Statistical analysis demonstrated that NFE2L3 expression was significantly higher in the invasive fronts and metastatic sites versus normal tissues $(P<0.001)$ (Figure 9B), consistent with its role in promoting invasion and progression.

\section{Discussion}

Patients with metastatic thyroid cancer, particularly individuals with small pulmonary metastases, typically enjoy a long progression-free survival before tumor growth occurs later, suggesting that metastatic dormancy is intrinsic to the majority of cases. These clinical data make thyroid cancer an excellent model to study mechanisms by which dormancy is maintained and/or lost. One such mechanism is the loss of metastasis-suppressing proteins. We have previously identified RCAN1-4 as a potentially important metas- 
A
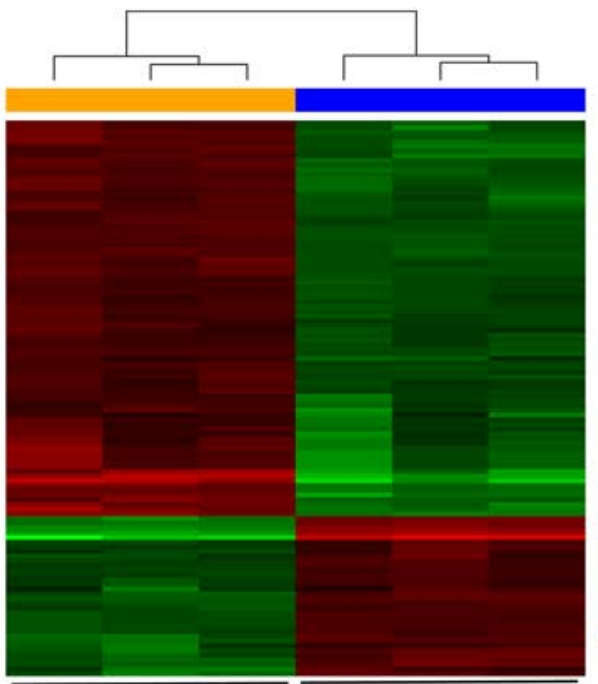

FTC236 shRCAN1-4 cells FTC236 shCtrl cells

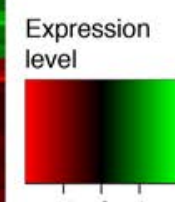

$\begin{array}{lll}-1 & 0 & 1\end{array}$

B

FTC236

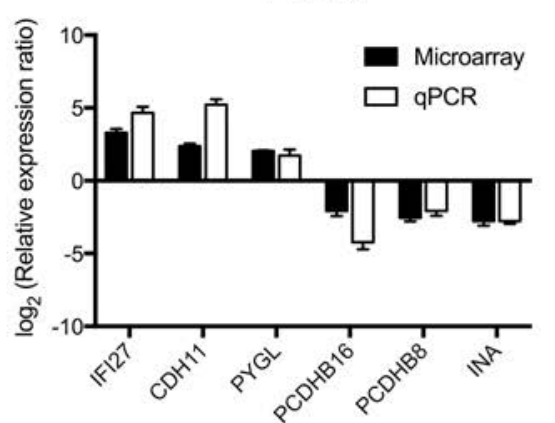

C

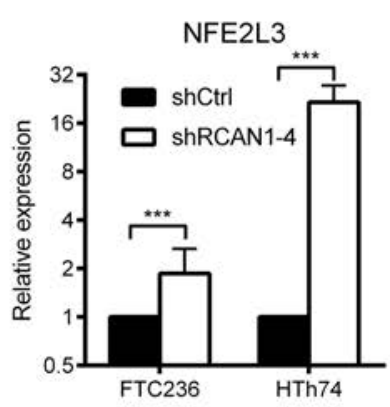

E

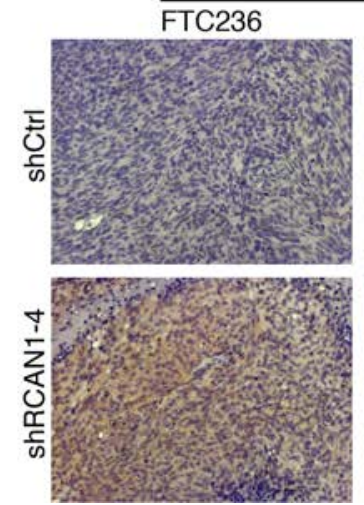

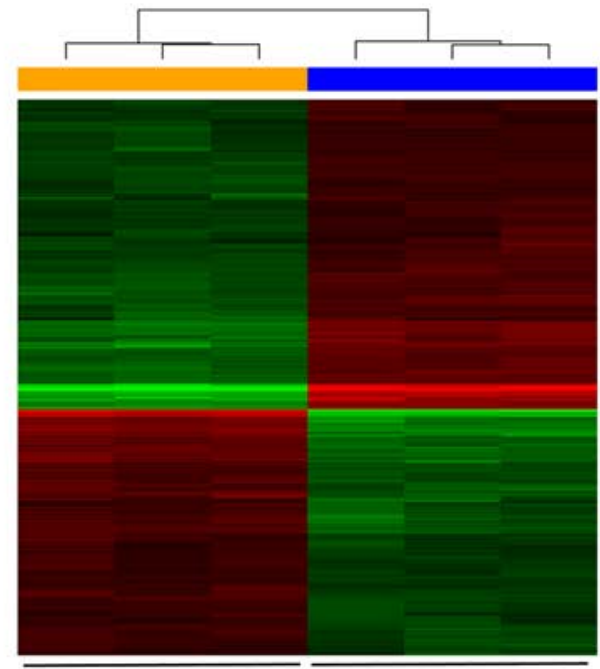

HTh74 shRCAN1-4 cells

HTh74

D
Expression

level

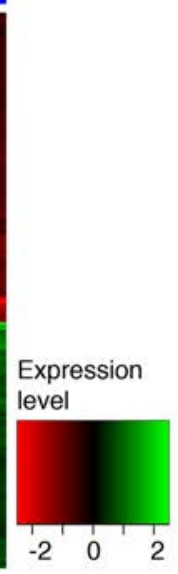

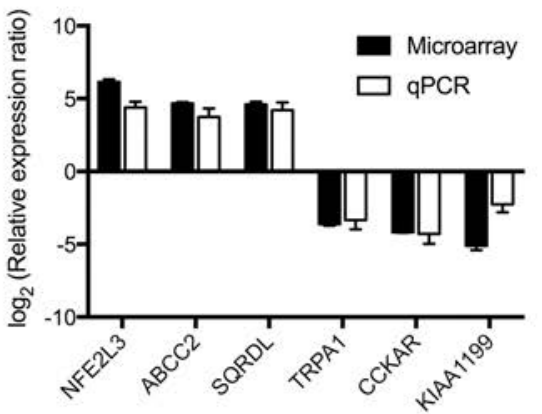

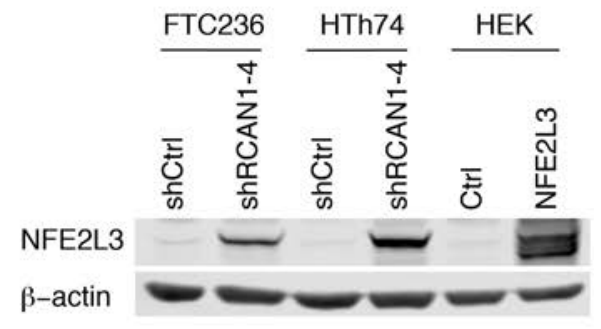

$\mathbf{F}$ HTh74
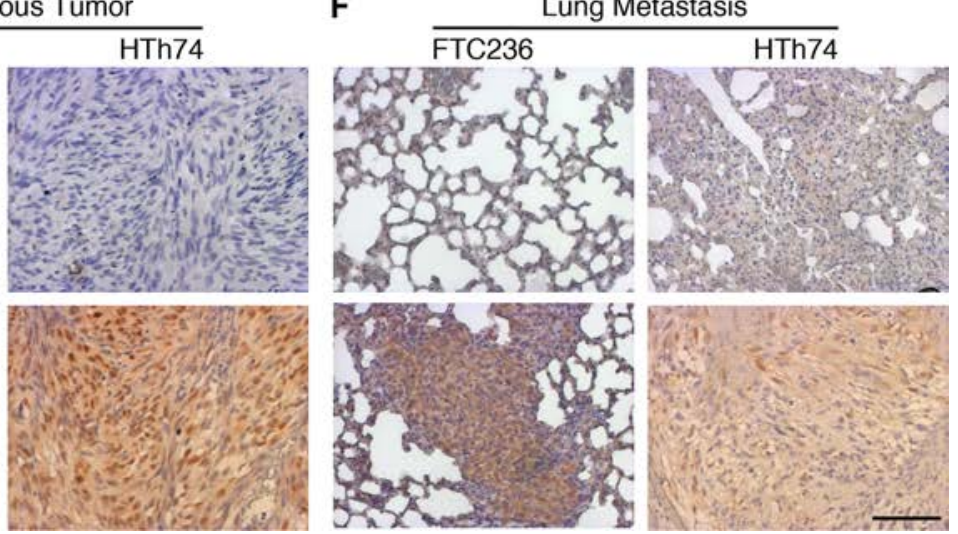

Figure 6. Microarray analysis of stable cell lines. (A) Heatmaps of the differentially expressed genes for FTC236 shCtrl cells versus shRCAN1-4 cells (left; 118 genes) and HTh74 shCtrl cells versus shRCAN1-4 cells (right; 420 genes). (B) Microarray data were validated by qRT-PCR of genes identified in PCA. The top 3 upregulated genes and top 3 downregulated genes from each cell line were chosen. Data are expressed as the log lalue $_{\text {val }}$ the relative expression levels. Error bars represent SEM of biological triplicates. (C) qRT-PCR results for in both cell lines. The HTh74 qRT-PCR plot was from the same data as in B. Bars represent SEM. A linear mixed model was used to determine the statistical significance. ${ }^{* *} P<0.001$. (D) Western blot analysis of the NFE2L3 expression level in the stable cells for both cell lines. (E and F) NFE2L3 IHC of the xenograft tumors (E) and lung metastases (F) demonstrated overexpression of NFE2L3 in shRCAN1-4 group. Scale bar: $100 \mu \mathrm{m}$.

tasis-suppressing protein based on in vitro work that identified it as a critical functional signaling node for cell invasion downstream of the metastin-GPR54 metastasis-suppressing signaling pathway (28). In these studies, RCAN1-4 loss and forced overexpression were reported to be important regulators of cell motility and invasion in thyroid cancer, melanoma, and colorectal cancer cell lines, implying a potent role in beyond thyroid cancer $(28,29)$. In the present study, we have extended this work in vivo, demonstrating that endogenous RCAN1-4 plays a vital role in suppressing tumor growth in xenografts and metastatic take rate and/ 
A

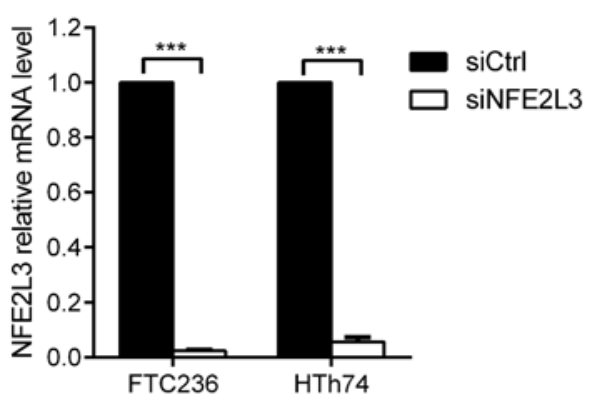

B

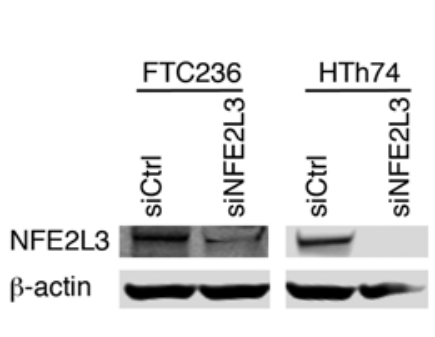

C

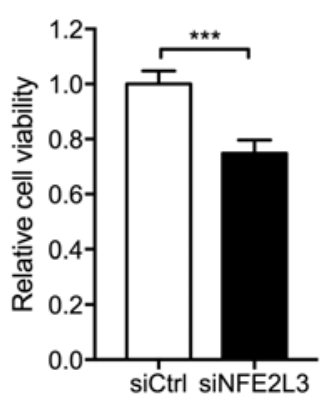

HTh74

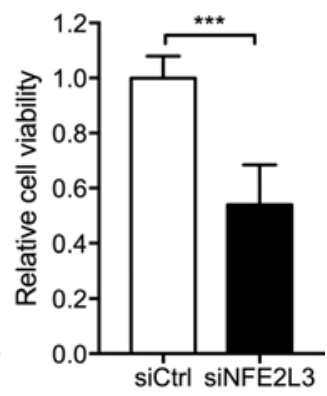

D

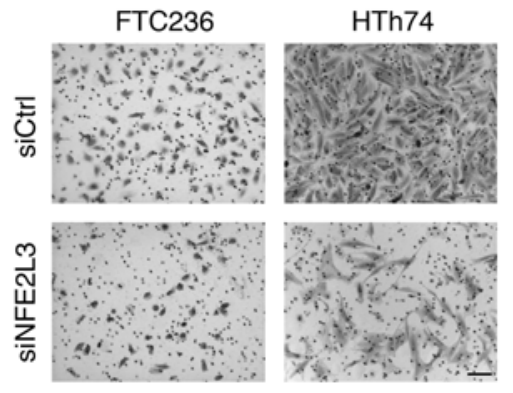

E

\section{FTC236}

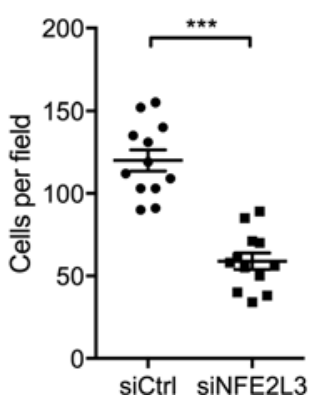

HTh74

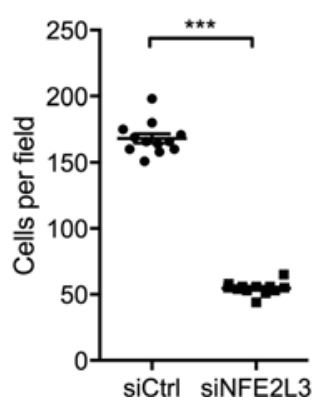

Figure 7. NEF2L3 knockdown suppresses cell invasion. (A and B) FTC and HTh74 shRCAN1-4 cells were transfected with either scrambled control siRNA (siCtrl) or NFE2L3 siRNA (siNFE2L3). Knockdown of NFE2L3 was confirmed using qRT-PCR (A) and Western blot (B). (C) The siCtrl-transfected and siNFE2L3-transfected cells were subjected to 3D spheroid growth assay. Cell viability was determined using the CellTiter-Clo 3D Cell Viability Kit. Data were normalized to the siCtrl-transfected cells. (D) Cell invasion images showed that siNFE2L3 transfection decreased cell invasion. Scale bar: $100 \mu \mathrm{m}$. (E) The number of invaded cells per field was quantified. Data points represent the number of invaded cells per field. The horizontal line represents the mean and the error bars represent the SEM. Linear mixed models were used to determine the statistical significance. ${ }^{* *} P<0.001$.

or growth in lungs in vivo. Furthermore, we identified NFE2L3 as a functional downstream effector of RCAN1-4 and indicated that its overexpression is associated with RCAN1-4 loss in vivo. Finally, NEF2L3 expression is increased in a large population of patients with thyroid cancer, and its overexpression is maintained or increased in thyroid cancer invasive fronts and distant metastases.

Using shRCAN1-4, we were able to reduce RCAN1-4 expression in two different thyroid cancer cell lines, FTC236 and HTh74 cells, with different genetic backgrounds. FTC236 has loss of Pten and a TP53 mutation and is derived from a recurrent lesion in a patient with FTC, while HTh74 is an ATC cell line characterized by TP53 mutation $(33,34)$. In both cases, the effects were similar with an increase in invasiveness and growth in $3 \mathrm{D}$ culture models in vitro and enhanced xenograft and lung metastases growth in vivo. It is of interest that the growth advantage that occurs with loss of RCAN1-4 was not identified in $2 \mathrm{D}$ culture. Indeed, many different conditions were attempted in $2 \mathrm{D}$ culture to determine whether other tumor-related changes were necessary for RCAN1-4 effects, including low glucose, hypoxia, and low or no serum, and no differences were identified (data not shown). By contrast, there was a distinct and statistically significant effect on growth in the spheroid 3D culture system. In many respects, 3D cell culture has more physiologically relevant characteristics in comparison to 2D systems and is believed to be more reflective of in vivo cell growth (35). This concept is supported by our data in which the 3D model system more closely matched the xenograft model results. These data, along with the Matrigel invasion data, suggest that RCAN1-4 effects may require an intracellular matrix to be present. We recognize that we used a single shRNA for these experiments, raising the possibility of nonspecific effects. In this small 299-base pair region, a total of 8 shRNAs were designed, but unfortunately only one reduced RCAN1-4 expression level. However, the in vitro data using this shRNA and the inducible overexpression in the present manuscript are consistent with our previously published data using transient siRNA and overexpression models (29) (Figure 5) and were consistent for two cell lines, thereby supporting specificity for RCAN1-4.

Interestingly, RCAN1-4 also has been reported to inhibit tumor growth when overexpressed in endothelial cells or the tumor microenvironment, with reduced vascularity $(24,26)$. These data, in addition to studies that defined a role for RCAN1-4 in the reduction of certain tumor types in Down's syndrome 
A
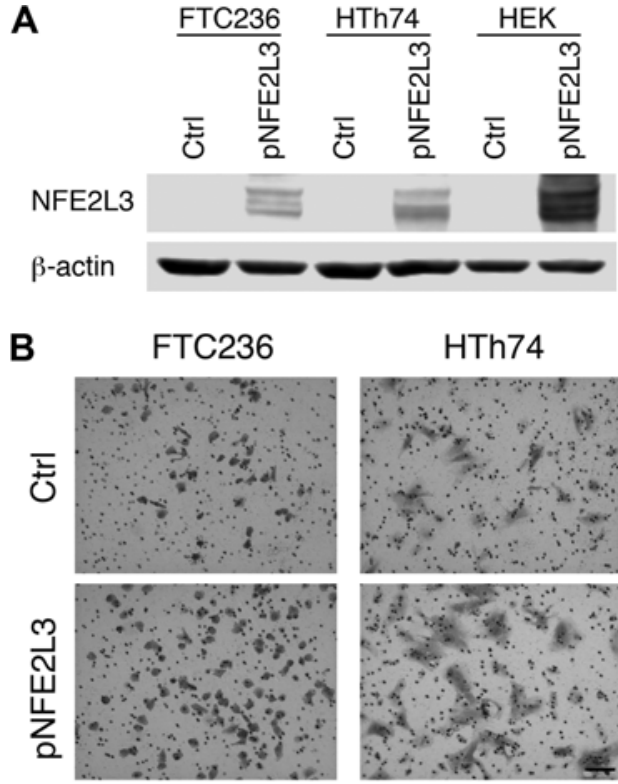

C

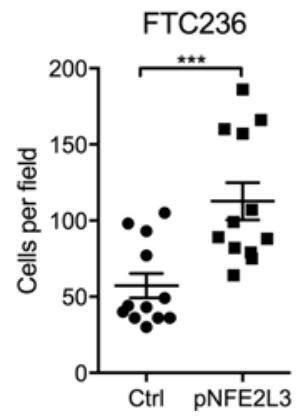

\section{HTh74}
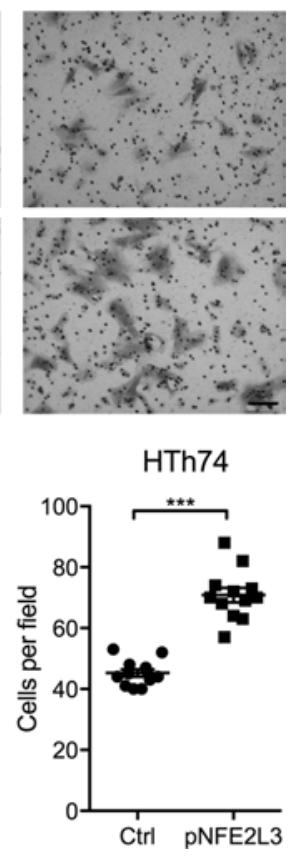

Figure 8. NFE2L3 overexpression increases cell invasion. (A) shCtrl cells were transfected with either control plasmid (Ctrl) or NFE2L3 plasmid (pNFE2L3). Western blot probed with NFE2L3 primary antibody demonstrated overexpression of NFE2L3 in both FTC236 and HTh74 cells. HEK 293 (HEK) cells were used as a positive control for transfection. (B) Cells were subjected to Matrigel invasion assay. Representative images of invaded cells are shown. Scale bar: $100 \mu \mathrm{m}$. (C) Cells from each field were counted. The experiments were repeated at least 3 times. Four fields were counted per experiment. Results are expressed as mean $\pm \mathrm{SEM}$. A linear mixed model was used to determine the statistical significance. ${ }^{* *} P<0.001$.

(trisomy 21) using murine models, suggest a potential role for RCAN1-4 in the tumor microenvironment (18). In the present study, we have focused on the cancer cell-autonomous effects of RCAN1-4 based on our prior in vitro work. A recent report confirms the importance of cancer cell RCAN1-4 by using array CGH to examine genetic alternations in chromosome 21, focusing on the sporadic forms of tumors most reduced in Down's syndrome patients (Wilms' tumor, lung cancer, breast cancer, and melanoma). The work found that RCAN1 gene deletion is the most common genetic aberration on chromosome 21 in Wilms' tumor, and it is also the only locus with a loss in all four cancer types examined, implicating a role for RCAN1 loss in these four tumors (27). Functional data were not included in that study.

While the data in the present study demonstrate that RCAN1-4 in cancer cells modulates tumor growth by promoting proliferation in vitro and in vivo, consistent with cancer cell-autonomous effects, a significant increase in the number of intratumoral microvessels in HTh74 shRCAN1-4 tumors was identified (Supplemental Figure 1). To our knowledge, this is the first time that RCAN1-4 in cancer cells has been reported to regulate tumor growth angiogenesis in vivo, and this suggests important interactions between the tumor and the host that may influence growth and progression. It is notable that there was no identifiable angiogenesis effect in the FTC236 shRCAN1-4 tumors, further suggesting that there may be cell line-specific effects. Thus, it may be that the lower frequency of specific solid tumor types in Down's syndrome is in part due to the effects of a third copy of RCAN1-4 in both the tumor cells and the tissues present in the microenvironment.

Metastatic progression is a highly coordinated process with a series of steps that involve complex interactions between cancer cells and the microenvironment. While we have demonstrated regulation of growth and invasion in vivo, the tail vein metastasis model provides evidence that RCAN1-4 is a regulator in the late steps of metastasis, such as extravasation, colonization, and/or secondary cell growth at metastatic sites. We have not yet tested the role of RCAN1-4 in the early steps of metastasis, and it is possible that the data may primarily reflect both growth- and metastasis-inhibiting effects, particularly in the Hth74 cell line that has dramatic growth inhibitory effects in the flank xenograft model. To further explore which step is regulated by RCAN1-4, studies using spontaneous metastatic tumor models and timed regulation of RCAN1-4 expression in immunocompetent models are needed. Such studies are ongoing but are beyond the scope of this current report.

In addition to indicating the effects of tumor cell RCAN1-4 on in vivo growth and metastasis, we also identified a mechanistic role for NFE2L3 in RCAN1-4-mediated effects. NFE2L3 is a member of the cap " $n$ " collar (CNC) family of basic leucine zipper transcription factors. CNC proteins, including NFE2L3, partner with Maf proteins to create complexes that bind Maf recognition elements and regulate gene transcription (36). NFE2L3 has been reported to be overexpressed in a variety of cancers and/or cancer cell lines, including lymphoma, testicular cancer, colorectal cancer, and breast cancer (37-40) and in large populations of patients with glioblastoma and lung and gastric adenocarcinomas (41-43). Indeed, TCGA gene expression data demonstrated that NFE2L3 is overexpressed by at least 2-fold in 17 types of cancer (44), which indicated that NFE2L3 might play a broad role across cancer types. However, its function in cancer is incompletely defined. In the present study, we demonstrate that NFE2L3 is overexpressed in thyroid cancer tissues, both at the mRNA level in TCGA primary PTC as well as in tumor and distant metastases from aggressive thyroid cancers, by IHC. Importantly, we provide evidence that NFE2L3 regulates cancer cell growth and invasion in vitro and that it is functionally important in RCAN1-4-mediated 3D cell growth and invasion. We also demonstrated that NFE2L3 is upregulated in tumors with loss of RCAN1-4 in vivo. The important functional downstream targets of NFE2L3 and the precise mechanisms by which RCAN1-4 regulates NFE2L3 expression are yet to be determined; however, the data demonstrate potentially important biological roles for NFE2L3 in cancer biology that warrant further study. 
A

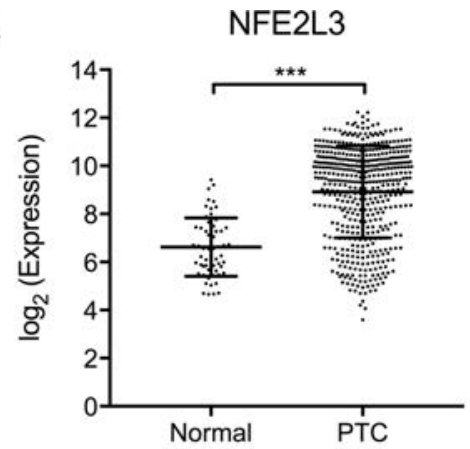

B

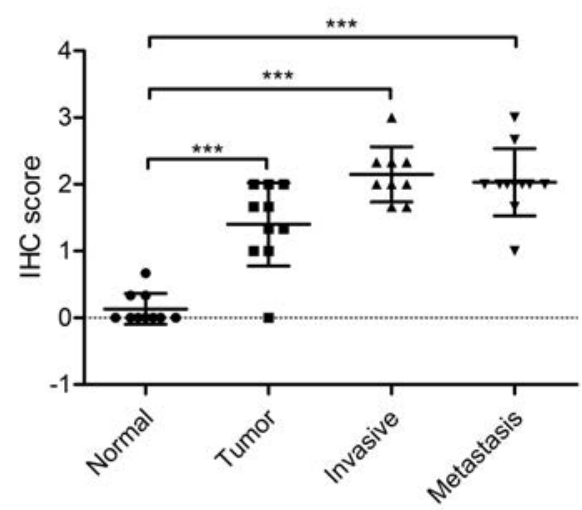

C

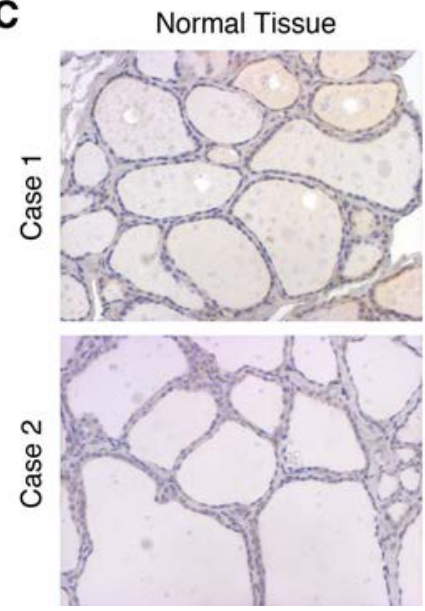

Tumor
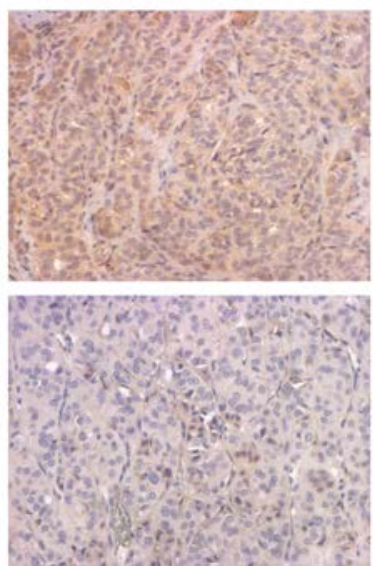

Invasive area
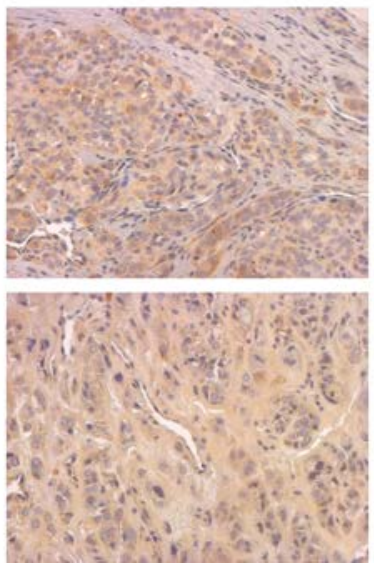
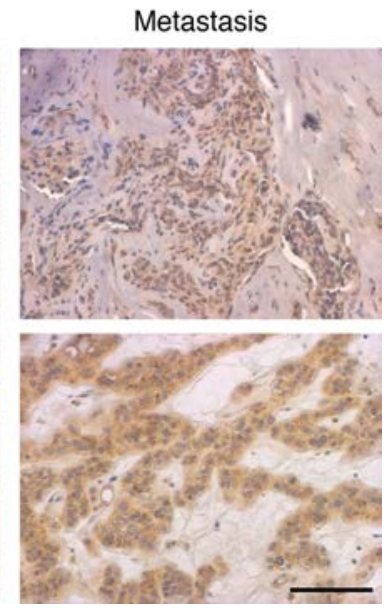

Figure 9. NFE2L3 is overexpressed in thyroid cancer samples. (A) NFE2L3 expression levels of 59 normal samples (Normal) and 503 papillary thyroid cancer samples (PTC) from TCGA thyroid cohort were plotted. Each point represents one sample. The horizontal lines represent mean \pm SEM. (B) NFE2L3 IHC staining was performed using tissue samples collected at The Ohio State University. See Supplemental Table 1 for detailed sample information. The IHC staining was scored blindly by three researchers on a 0-3 scale based on staining intensity. The scores corresponding to normal tissue (Normal), tumor center (Tumor), tumor invasive front (Invasive), and metastatic sites (Metastasis) were plotted. Each data point is one sample. The horizontal lines represent mean \pm SEM. (C) Representative IHC images from two cases of FTC demonstrate increasing NFE2L3 expression. Scale bar: $100 \mu \mathrm{m}$. For TCGA data (A), 1-way ANOVA was used for analysis. For IHC staining data (B), a linear mixed model was used for analysis and Holm's procedure was used for controlling multiple comparisons. ${ }^{* *} P<0.001$.

In summary, we provide evidence that RCAN1-4 in cancer cells regulates tumor growth and metastasis in vivo. We demonstrate that the mechanism of RCAN1-4 effects involves NFE2L3 and further suggest that this transcription factor may be an important and previously unidentified regulator of cell invasion. NFE2L3 is overexpressed in a number of cancer types, including thyroid cancer, and it is overexpressed in the invasive fronts of primary tumors and metastatic tissues in thyroid cancer. This warrants further study of NFE2L3 as a potential regulator of cancer progression.

\section{Methods}

Cell culture. Human thyroid cancer cell lines 8505c, BCPAP, C643, FTC236, and SW1736 were gifts from Rebecca Schweppe and Bryan Haugen (University of Colorado, Denver, Colorado, USA), with permission from originating laboratories where the cells were established. All the cells were confirmed to be of thyroid origin by short tandem repeat profiling as previously described (45). FTC236 cells were grown in DMEM (Invitrogen). All other cell lines were grown in RPMI 1640 (Invitrogen). All media were supplemented with $10 \%$ fetal bovine serum (Invitrogen) and non-essential amino acids (Invitrogen). The cells were maintained at $37^{\circ} \mathrm{C}$ and $5 \% \mathrm{CO}_{2}$ in a humidified incubator.

Generation of stable cell lines. FTC236-luciferase cells, C643-luciferase (C643-1uc) cells, and SW1736-luciferase cells were established by transfecting the cells with pCDNA3.1 (+) yellow fluorescence proteinluciferase reporter plasmid (a gift from Thomas J. Rosol, The Ohio State University) (46) with Amaxa 
Nucleofector (Lonza) and selected with G418 (Sigma-Aldrich) for 2 weeks. The resistant clones were further screened for luciferase activity using the Luciferase Assay System (Promega). HTh74 luciferase cells, BCPAP luciferase (BCPAP-luc) cells, and 8505c luciferase cells were gifts from Rebecca Schweppe (University of Colorado) (47). The FTC236-luc and HTh74-luc cells were transfected with nontargeting scrambled shCtrl or shRCAN1-4 (OriGene) and selected with $400 \mathrm{ng} / \mathrm{ml}$ and $300 \mathrm{ng} / \mathrm{ml}$ puromycin (Invitrogen), respectively, for 2 weeks. RCAN1-4 knockdown clones were screened using Western blot. 8505c-luc cells and C643-luc cells were transfected with pcDNA3.1 (+) - rtTA vector (Clontech) and selected with $200 \mu \mathrm{g} / \mathrm{ml}$ and $400 \mu \mathrm{g} / \mathrm{ml}$ zeocin, respectively, for 2 weeks to generate the $8505 \mathrm{c}-\mathrm{rtTA}$ and C643-rtTA cells. The 8505c-rtTA and C643-rtTA cells were transfected with pTRE-RCAN1-4 construct and selected with $1.5 \mu \mathrm{g} / \mathrm{ml}$ and $200 \mathrm{ng} / \mathrm{ml}$ puromycin, respectively, for 2 weeks to generate the $8505 \mathrm{c}$ rtTA TRE-RCAN1-4 cells and C643 rtTA TRE-RCAN1-4 cells.

Western blot and antibodies. Protein isolation and Western blot were performed as previously described (48). Primary antibodies against RCAN1 (1:1,000 dilution, catalog D6694) and NFE2L3 (1:1,000 dilution, catalog HPA055889) were obtained from Sigma-Aldrich. Antibody against $\beta$-actin (1:1,000 dilution, catalog sc-8432) was obtained from Santa Cruz. See complete unedited blots in the supplemental material.

$R N A$ isolation and $q R T-P C R$. Total RNA was isolated using TRIzol (Invitrogen) according to the manufacturer's instructions. RNA was treated with DNase I (Invitrogen) for 15 minutes. Reverse transcription was performed using MultiScribe reverse transcriptase (Applied Biosystems) following the manufacturer's protocol. qRT-PCR was performed using SYBR Green (Applied Biosystems) for RCAN1-4 and TaqMan assay (Applied Biosystems) for NFE2L3 and other targets. 18s rRNA was used as the internal control.

$2 \mathrm{D}$ and $3 \mathrm{D}$ cell growth assay. $2 \mathrm{D}$ cell growth assay was carried out as previously described (49). Basically, cells $\left(0.5 \times 10^{5}\right.$ for FTC236 or $1 \times 10^{5}$ for HTh74) were seeded in 6-well plates in triplicate in cell growth medium. Viable cells were counted using the Countess Automated Cell Counter (Invitrogen) at 24, 48, and 72 hours after seeding. For 3D cell growth assay, 1,000 cells were seeded in 96-well spheroid microplates (Corning). Cell viability was assessed using CellTiter-Glo 3D Cell Viability Assay (Promega).

Cell transfection. Cells were seeded in $10-\mathrm{cm}$ dishes at about $70 \%$ confluency. For siRNA transfection, cells were transfected with 500 picomoles of siRNA against NFE2L3 or scrambled control siRNA (Santa Cruz) using Lipofectamine 2000 (Invitrogen) following the manufacturer's protocol. For plasmid transfection, cells were transfected with $4 \mu \mathrm{g}$ pcDNA3.1/ Hygro (+) - NFE2L3 plasmid (a gift from Volker Blank, McGill University, Montreal, Quebec, Canada) (30) using Optifect Reagent (Invitrogen). Cells were incubated with the liposome complex for 4 hours before switched to normal growth medium.

Cell invasion assay. Cell invasion assay was performed using the Corning BioCoat Growth Factor Reduced (GFR) Matrigel Invasion Chambers (Corning) following the manufacturer's protocol. Cells were collected and resuspended in medium with no serum containing $0.1 \%$ BSA. Cells $\left(5 \times 10^{4}\right.$ in $500 \mu 1$ medium $)$ were seeded in the rehydrated GFR Matrigel inserts with $8.0-\mu \mathrm{m}$ pores. The inserts were transferred into wells with DMEM containing 1\% FBS or RPMI containing 10\% FBS as the chemoattractant for FTC236 cells and $\mathrm{HTh} 74$ cells, respectively. The cells were then incubated at $37^{\circ} \mathrm{C}, 5 \% \mathrm{CO}_{2}$ for 24 hours for FTC236 cells and 36 hours for HTh74 cells. At the end of the experiments, cells were washed with PBS containing calcium and magnesium, fixed with $3.7 \%$ formaldehyde containing $0.05 \%$ crystal violet for 30 minutes, and washed with distilled water. Pictures of cells on (noninvaded) and under (invaded) the membranes were taken using an Axiovert 40 CFL microscope (Zeiss) (50). Invaded cells were counted using the ImageJ software (NIH).

Live-animal imaging. A Lumina II Imaging System (Perkin Elmer) was used to measure the bioluminescence from mice. $150 \mathrm{mg}$ D-luciferin (Gold Biotechnology) per kilogram of body weight was injected into mice intraperitoneally. Bioluminescence was measured 10 minutes after D-luciferin injection at the Small Animal Imaging Core at The Ohio State University. Living Image 4.5.2 (Perkin Elmer) was used to quantify the tumor bioluminescence signal for the subcutaneous model and mouse whole body signal for the tail vein metastasis model.

Subcutaneous injection model. $1 \times 10^{6}$ cells were collected and resuspended in $100 \mu \mathrm{PBS}$ and mixed with 100 $\mu 1$ Matrigel. Athymic nude mice (4-5 weeks old, obtained from Target Validation Shared Resources at The Ohio State University) were anesthetized using isoflurane (Henry Schein). The cell and Matrigel mixture was injected subcutaneously into the ventrolateral flank of mice. Tumors were measured weekly using both caliper and IVIS Lumina II In Vivo Imaging System (PerkinElmer). Histology staining was performed for the tumors and lungs after mice euthanasia at the end of the study. Tumor volumes were calculated using the following formula: tumor volume $=1 / 2 \times($ length $\times$ width $\times$ width $)(51)$. 
Tail vein metastasis model. $1.5 \times 10^{6}$ cells were resuspended in PBS and injected into the tail veins of athymic nude mice using 27-gauge insulin syringes (Terumo). Mice were monitored weekly using IVIS to detect metastases. Successful injections resulting in cells in the lung circulation were confirmed by lung bioluminescence signals upon immediate IVIS imaging after injection. Mice were sacrificed 5 weeks after injection for FTC236 cells and 12 weeks after injection for HTh74 cells. Lung tissues and other bioluminescence-positive tissues were collected and subjected to histology staining and examination.

Histology and IHC. Tumors and tissues were fixed in 10\% zinc formalin (Thermo Scientific) for 3 days and then paraffin embedded and sectioned $(4 \mu \mathrm{m})$. H\&E, Ki-67 (1:200 dilution, Abcam, catalog ab16667), CD31 (1:250 dilution, Santa Cruz, catalog sc-1506R), and cleaved caspase-3 (1:800 dilution, Cell Signaling, catalog 9661) staining were performed by the Solid Tumor Pathology Core at The Ohio State University as previously described (52). F4/80 staining was performed by the Comparative Pathology and Mouse Phenotyping Shared Resources at The Ohio State University. NFE2L3 staining (1: 50 dilution, Sigma-Aldrich, catalog HPA055889) was performed using a previously published protocol (53). To quantify the IHC staining, 10 high-power field images $(\times 20)$ were taken for each slide using a Vectra imaging system (Perkin Elmer) and averaged for each case. Only cells with a whole nucleus were taken into account, and only intratumoral areas were used for quantification. Quantification of IHC staining and microvessels was performed automatically using inForm software (version 2.0.2) (Perkin Elmer). Positivity was expressed as a percentage of positively stained cells relative to the total number of cells visualized by hematoxylin counterstain. Microvessel density quantification was performed following previously described criteria (54).

Microarray. RNA from shCtrl and shRCAN1-4 cells was isolated using the Total RNA Purification Kit (Norgen Biotek) following manufacturer's protocol. The RNA was processed and applied to GeneChip Human Transcriptome Array 2.0 (Affymetrix) by the Genomics Shared Resource at The Ohio State University. Signal intensities were processed by the RMA method using Affymetrix Expression Console software (55). A filtering method based on the percentage of arrays above noise cutoff was applied to filter out low expression genes. Linear model was employed to detect differentially expressed genes between conditions. In order to improve the estimates of variability and statistical tests for differential expression, a variance smoothing method with moderated t-statistic was employed for this study (56). The significance level was adjusted by controlling the mean number of false positives (57). Heatmap with hierarchical clustering and PCA were applied to explore the data. Statistical software SAS 9.4 (SAS Institute Inc.) and R were used for analysis. Raw data were deposited at NCBI's GEO (https://www.ncbi.nlm.nih.gov/geo/) with accession GSE93250.

Statistics. The tumor growth and metastasis data were $\log _{10}$ transformed to reduce variance and skewness. ANOVA was used to analyze the metastasis experiment. Linear mixed effects models were used to analyze tumor growth and invasion assay data to take into account the correlations among observations from the same animal measured over time or observations obtained on the same day. $P<0.05$ was considered significant. SAS 9.4 (SAS Institute Inc.) was used for all the analyses.

Study approval. The patient sample collection procedures were approved by The Ohio State University Institutional Review Boards, and patients' written consents were obtained prior to inclusion in the study. All animal studies were approved by The Ohio State University Institutional Animal Care and Use Committee.

\section{Author contributions}

MS and MDR designed the study; CW, MS, AMY, HN, and NP conducted the experiments; CW, SEJ, XZ, LY, SF, PW, KLP, MS, and MDR analyzed the data; and CW and MDR wrote the manuscript.

\section{Acknowledgments}

This work is supported by NIH grant R01 CA152066 to MDR and NIH cancer center grant P30 CA016058 to the following shared resources: Biostatistics, Comparative Pathology and Mouse Phenotyping, Genomics, and Small Animal Imaging Core. CW is also supported by The Ohio State Pelotonia Graduate Fellowship and the Presidential Fellowship. We would like to acknowledge N. Denko for assistance and input with the manipulations of 2D cell culture systems and V. Blank for generously sharing NFE2L3 expression vectors.

Address correspondence to: Matthew D. Ringel, 565 McCampbell Hall, 1581 Dodd Drive, Columbus, Ohio 43210, USA. Phone: 614.6853333; E-mail: matthew.ringel@osumc.edu. 
1. Nguyen DX, Bos PD, Massagué J. Metastasis: from dissemination to organ-specific colonization. Nat Rev Cancer. 2009;9(4):274-284

2. Stafford LJ, Vaidya KS, Welch DR. Metastasis suppressors genes in cancer. Int J Biochem Cell Biol. 2008;40(5):874-891.

3. Steeg PS, et al. Evidence for a novel gene associated with low tumor metastatic potential. J Natl Cancer Inst. 1988;80(3):200-204.

4. Rinker-Schaeffer CW, O'Keefe JP, Welch DR, Theodorescu D. Metastasis suppressor proteins: discovery, molecular mechanisms, and clinical application. Clin Cancer Res. 2006;12(13):3882-3889.

5. Ozturk S, et al. SDPR functions as a metastasis suppressor in breast cancer by promoting apoptosis. Proc Natl Acad Sci USA. 2016;113(3):638-643.

6. Montagner M, et al. SHARP1 suppresses breast cancer metastasis by promoting degradation of hypoxia-inducible factors. Nature. 2012;487(7407):380-384.

7. Horak CE, Lee JH, Marshall JC, Shreeve SM, Steeg PS. The role of metastasis suppressor genes in metastatic dormancy. APMIS. 2008;116(7-8):586-601.

8. Okugawa Y, et al. Loss of the metastasis suppressor gene KiSS1 is associated with lymph node metastasis and poor prognosis in human colorectal cancer. Oncol Rep. 2013;30(3):1449-1454.

9. Dong JT, et al. Down-regulation of the KAI1 metastasis suppressor gene during the progression of human prostatic cancer infrequently involves gene mutation or allelic loss. Cancer Res. 1996;56(19):4387-4390.

10. Giancotti FG. Mechanisms governing metastatic dormancy and reactivation. Cell. 2013;155(4):750-764.

11. Ringel MD. Metastatic dormancy and progression in thyroid cancer: targeting cells in the metastatic frontier. Thyroid. 2011;21(5):487-492.

12. Sherman SI, et al. Prospective multicenter study of thyroiscarcinoma treatment: initial analysis of staging and outcome. National Thyroid Cancer Treatment Cooperative Study Registry Group. Cancer. 1998;83(5):1012-1021.

13. Durante C, et al. Long-term outcome of 444 patients with distant metastases from papillary and follicular thyroid carcinoma: benefits and limits of radioiodine therapy. J Clin Endocrinol Metab. 2006;91(8):2892-2899.

14. Xavier AC, Ge Y, Taub JW. Down syndrome and malignancies: a unique clinical relationship: a paper from the 2008 william beaumont hospital symposium on molecular pathology. J Mol Diagn. 2009;11(5):371-380.

15. Hasle H, Clemmensen IH, Mikkelsen M. Risks of leukaemia and solid tumours in individuals with Down's syndrome. Lancet. 2000;355(9199):165-169.

16. Satgé D, et al. A lack of neuroblastoma in Down syndrome: a study from 11 European countries. Cancer Res. 1998;58(3):448-452.

17. Yang Q, Rasmussen SA, Friedman JM. Mortality associated with Down's syndrome in the USA from 1983 to 1997: a population-based study. Lancet. 2002;359(9311):1019-1025.

18. Baek KH, et al. Down's syndrome suppression of tumour growth and the role of the calcineurin inhibitor DSCR1. Nature. 2009;459(7250):1126-1130.

19. Davies KJ, et al. Renaming the DSCR1/Adapt78 gene family as RCAN: regulators of calcineurin. FASEB J. 2007;21(12):3023-3028.

20. Jang C, Lim JH, Park CW, Cho YJ. Regulator of calcineurin 1 isoform 4 (RCAN1.4) Is overexpressed in the glomeruli of diabetic mice. Korean J Physiol Pharmacol. 2011;15(5):299-305.

21. Martínez-Martínez S, et al. The RCAN carboxyl end mediates calcineurin docking-dependent inhibition via a site that dictates binding to substrates and regulators. Proc Natl Acad Sci USA. 2009;106(15):6117-6122.

22. Hogan PG, Chen L, Nardone J, Rao A. Transcriptional regulation by calcium, calcineurin, and NFAT. Genes Dev. 2003;17(18):2205-2232.

23. Mancini M, Toker A. NFAT proteins: emerging roles in cancer progression. Nat Rev Cancer. 2009;9(11):810-820.

24. Minami T, et al. Vascular endothelial growth factor- and thrombin-induced termination factor, Down syndrome critical region-1, attenuates endothelial cell proliferation and angiogenesis. J Biol Chem. 2004;279(48):50537-50554.

25. Iizuka M, Abe M, Shiiba K, Sasaki I, Sato Y. Down syndrome candidate region 1, a downstream target of VEGF, participates in endothelial cell migration and angiogenesis. J Vasc Res. 2004;41(4):334-344.

26. Minami T, et al. The Down syndrome critical region gene 1 short variant promoters direct vascular bed-specific gene expression during inflammation in mice. J Clin Invest. 2009;119(8):2257-2270.

27. Forés-Martos J, Cervera-Vidal R, Chirivella E, Ramos-Jarero A, Climent J. A genomic approach to study Down syndrome and cancer inverse comorbidity: untangling the chromosome 21. Front Physiol. 2015;6:10.

28. Stathatos N, et al. KiSS-1/G protein-coupled receptor 54 metastasis suppressor pathway increases myocyte-enriched calcineurin interacting protein 1 expression and chronically inhibits calcineurin activity. J Clin Endocrinol Metab. 2005;90(9):5432-5440.

29. Espinosa AV, et al. Regulator of calcineurin 1 modulates cancer cell migration in vitro. Clin Exp Metastasis. 2009;26(6):517-526.

30. Kannan MB, Dodard-Friedman I, Blank V. Stringent control of NFE2L3 (nuclear factor, erythroid 2-like 3; NRF3) protein degradation by FBW7 (F-box/WD repeat-containing protein 7) and glycogen synthase kinase 3 (GSK3). J Biol Chem. 2015;290(43):26292-26302.

31. Broad Institute TCGA Genome Data Analysis Center (2016): Correlation between mRNAseq expression and clinical features. Broad Institute of MIT and Harvard. http://gdac.broadinstitute.org/runs/analyses_latest/reports/cancer/THCA/Correlate_ Clinical_vs_mRNAseq/nozzle.html. Accessed February 3, 2017.

32. Cancer Genome Atlas Research Network. Integrated genomic characterization of papillary thyroid carcinoma. Cell. 2014;159(3):676-690.

33. Goretzki PE, Frilling A, Simon D, Roeher HD. Growth regulation of normal thyroids and thyroid tumors in man. Recent Results Cancer Res. 1990;118:48-63.

34. Heldin NE, Cvejić D, Smeds S, Westermark B. Coexpression of functionally active receptors for thyrotropin and platelet-derived growth factor in human thyroid carcinoma cells. Endocrinology. 1991;129(4):2187-2193.

35. Edmondson R, Broglie JJ, Adcock AF, Yang L. Three-dimensional cell culture systems and their applications in drug discovery and cell-based biosensors. Assay Drug Dev Technol. 2014;12(4):207-218.

36. Kobayashi A, et al. Molecular cloning and functional characterization of a new Cap'n' collar family transcription factor Nrf3. J Biol Chem. 1999;274(10):6443-6452.

37. Chevillard G, Blank V. NFE2L3 (NRF3): the Cinderella of the Cap'n'Collar transcription factors. Cell Mol Life Sci. 2011;68(20):3337-3348. 
38. Chiu ST, Hsieh FJ, Chen SW, Chen CL, Shu HF, Li H. Clinicopathologic correlation of up-regulated genes identified using cDNA microarray and real-time reverse transcription-PCR in human colorectal cancer. Cancer Epidemiol Biomarkers Prev. 2005;14(2):437-443

39. Almstrup K, et al. Genomic and gene expression signature of the pre-invasive testicular carcinoma in situ. Cell Tissue Res. 2005;322(1):159-165.

40. Rhee DK, Park SH, Jang YK. Molecular signatures associated with transformation and progression to breast cancer in the isogenic MCF10 model. Genomics. 2008;92(6):419-428.

41. Cancer Genome Atlas Research Network. Comprehensive genomic characterization defines human glioblastoma genes and core pathways. Nature. 2008;455(7216):1061-1068.

42. Cancer Genome Atlas Research Network. Comprehensive molecular characterization of gastric adenocarcinoma. Nature. 2014;513(7517):202-209.

43. Cancer Genome Atlas Research Network. Comprehensive molecular profiling of lung adenocarcinoma. Nature. 2014;511(7511):543-550.

44. Broad Institute TCGA Genome Data Analysis Center (2016). Firebrowse Gene Expression Viewer. http://firebrowse.org/viewGene.html?gene=NFE2L3. TCGA data version 2016_01_28. Accessed February 3, 2017.

45. Schweppe RE, et al. Deoxyribonucleic acid profiling analysis of 40 human thyroid cancer cell lines reveals cross-contamination resulting in cell line redundancy and misidentification. J Clin Endocrinol Metab. 2008;93(11):4331-4341.

46. LeRoy BE, et al. New bone formation and osteolysis by a metastatic, highly invasive canine prostate carcinoma xenograft. Prostate. 2006;66(11):1213-1222.

47. Chan CM, et al. Targeted inhibition of Src kinase with dasatinib blocks thyroid cancer growth and metastasis. Clin Cancer Res. 2012;18(13):3580-3591.

48. Ma Y, et al. Development of p21 activated kinase-targeted multikinase inhibitors that inhibit thyroid cancer cell migration. $J$ Clin Endocrinol Metab. 2013;98(8):E1314-E1322.

49. Plews RL, et al. A novel dual AMPK activator/mTOR inhibitor inhibits thyroid cancer cell growth. J Clin Endocrinol Metab. 2015;100(5):E748-E756.

50. McCarty SK, et al. Group I p21-activated kinases regulate thyroid cancer cell migration and are overexpressed and activated in thyroid cancer invasion. Endocr Relat Cancer. 2010;17(4):989-999.

51. Faustino-Rocha A, et al. Estimation of rat mammary tumor volume using caliper and ultrasonography measurements. $L a b$ $\operatorname{Anim}(N Y)$. 2013;42(6):217-224

52. Caserta E, et al. Noncatalytic PTEN missense mutation predisposes to organ-selective cancer development in vivo. Genes Dev. 2015;29(16):1707-1720.

53. Saji M, et al. Akt1 deficiency delays tumor progression, vascular invasion, and distant metastasis in a murine model of thyroid cancer. Oncogene. 2011;30(42):4307-4315.

54. Weidner N, Semple JP, Welch WR, Folkman J. Tumor angiogenesis and metastasis--correlation in invasive breast carcinoma. $N$ Engl J Med. 1991;324(1):1-8.

55. Irizarry RA, et al. Exploration, normalization, and summaries of high density oligonucleotide array probe level data. Biostatistics. 2003;4(2):249-264.

56. Yu L, Gulati P, Fernandez S, Pennell M, Kirschner L, Jarjoura D. Fully moderated T-statistic for small sample size gene expression arrays. Stat Appl Genet Mol Biol. 2011;10(1):42.

57. Gordon A, Glazko G, Qiu X, Yakovlev A. Control of the mean number of false discoveries, bonferroni and stability of multiple testing. Ann Appl Stat. 2007;1(1):179-190. 